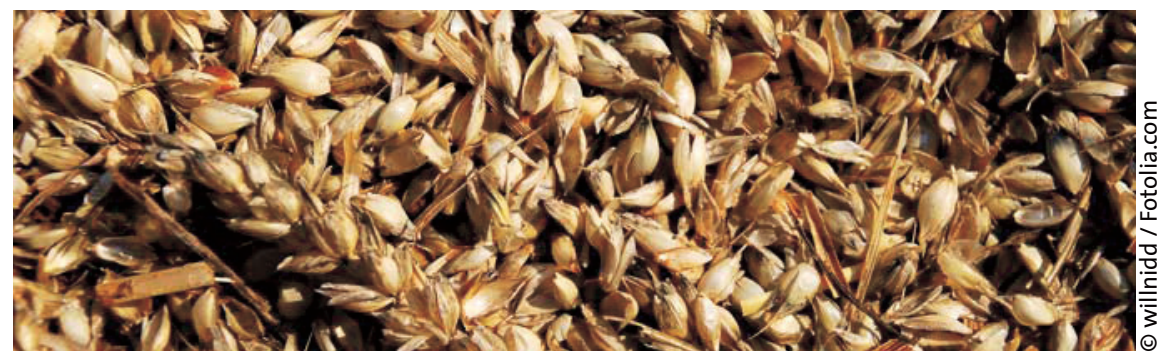

\section{Allergoide - die Spreu vom Weizen trennen}

$\mathrm{N}$ eben nativen Allergenen werden bei der spezifischen Immuntherapie (SIT) auch Allergoide eingesetzt. Bei diesen wird durch chemische Modifizierung die räumliche Struktur verändert und so die Anzahl der IgE-bindenden BZell-Epitope verringert, während die für die immunogene Wirkung verantwortlichen T-Zell-Epitope erhalten bleiben. Um eine evidenzbasierte Therapie zu gewährleisten, sollte aber ein Präparat verwendet werden, dessen Wirksamkeit durch methodisch geeignete Studien dokumentiert sei, empfahl Prof. Dr. Claus Bachert aus Gent.

Er präsentierte das Ergebnis einer Auswertung der entsprechenden wissenschaftlichen Literatur durch sein Team: „Die Evidenz zur klinischen Wirksamkeit entsprechend den Qualitätsanforderungen der $\mathrm{WHO}$ ist für Allergoide auf wenige Präparate begrenzt." Bei Gräserallergoiden wurden drei Studien mit drei verschiedenen Präparaten mit positivem Wirksamkeitsnachweis ermittelt, für Bäume eine Studie mit einem Präparat und für die Gruppe der Milbenallergoide zwei Studien zu einem Präparat. Ein Beispiel hob Bachert hervor: „Pollinex ${ }^{\circledR}$ Quattro ist ein Allergoidpräparat mit nachgewiesener Wirksamkeit bei Gräsern, Roggen und Baumpollen." Es enthält als Wirkverstärker das Adjuvans Monophosphoryl-Lipid A $\left(\mathrm{MPL}^{\circledR}\right)$, ein detoxifiziertes Lipid-A-Derivat aus der Zellmembran von Salmonella minnesota, das durch die Bindung an Toll-likeRezeptoren die körpereigene Immunabwehr und das immunologische Gedächtnis stimuliert. Für eine optimierte Formulierung des adjuvantierten Allergoids - 0,5 statt $1 \mathrm{ml}$ pro Injektion - zeigte jüngst eine multizentrische Studie mit über 1.000 Patienten, dass in der Population der Non-Missing-Cases $(\mathrm{n}=343)$ der kombinierte Symptom-MedikationsScore um 26,9\% $(\mathrm{p}=0,0031)$ im Vergleich zur Plazebogruppe reduziert werden konnte.

Über Erfahrungen mit Pollinex ${ }^{\circledR}$ Quattro in der präsaisonalen KurzzeitSIT mit nur vier Injektionen bei Kindern und Jugendlichen berichtete Prof. Dr. Stefan Zielen aus Frankfurt/Main. Aus ethischen Gründen sind die Möglichkeiten zur Datengewinnung bei dieser Gruppe eingeschränkt. Eine eigene Untersuchung an 43 Patienten im medianen Alter von elf Jahren ergab immerhin, dass vor allem im zweiten und dritten Jahr der Therapie ein signifikanter Anstieg der blockierenden IgGund $\mathrm{IgG}_{4}$-Antikörper als Surrogatmarker einer starken Immuntoleranz nachweisbar ist.

Dr. Uta Rabe aus Treuenbrietzen interessierte sich besonders für den Parameter, der ihren Patienten am wichtigsten ist: die Lebensqualität. Nach einer Erhebung mittels Fragebögen zu Beginn, Mitte und Ende der drei Therapiezyklen einer Kurzzeit-SIT bei 112 erwachsenen Baumpollenallergikern im Rahmen einer multizentrischen Anwendungsbeobachtung verminderten sich die Summenscores der Lebensqualität bei Rhinokonjunktivitis (RQLQ) um bis zu 44\% und bei Asthma (AQLQ) um bis zu $54 \%$. „Eine deutliche Verbesserung", so Rabes Resümee. Dafür nähmen die Patienten in einem dünn besiedelten Gebiet sogar längere Anfahrtswege zum Allergologen in Kauf die Compliance sei sehr gut.

we

Industriesymposium „Klinische Evidenz in der SIT“im Rahmen des 5. Deutschen Allergiekongresses. Hannover,

10. September 2010. Veranstalter: Bencard Allergie, München
Neues Anstiasthmatikum ante portas

Das Unternehmen Mundipharma hat eine neue Fixkombination zur Therapie des Asthma bronchiale in mehreren europäischen Staaten zur Zulassung eingereicht. Das Präparat wurde in Kooperation mit SkyePharma entwickelt und vereint die Vorteile des in Europa am häufigsten verschriebenen inhalativen Glukokortikoids Fluticasonproprionat mit denen des lang wirksamen $\beta_{2}$-Agonisten Formoterolfumarat, der sich durch einen raschen Wirkungseintritt auszeichnet.

Aktuelle Studiendaten zeigen, dass die neue Fixkombination genauso effektiv ist wie das zu den europäischen Marktführern zählende Duo Fluticasonproprionat/Salmeterolxinofoat, die Wirkung jedoch rascher eintritt. Darüber hinaus konnte belegt werden, dass die Kombination der beiden Wirkstoffe eine effektivere Kontrolle der Asthmasymptome ermöglicht als Fluticasonproprionat allein.

Nach Informationen von Mundipharma, Limburg

\section{Lob für Innovation}

Basilea Pharmaceutica erhielt im September zum zweiten Mal in Folge die „Goldene Tablette" für das beliebteste Pharmaunternehmen und mit Toctino $^{\circledR}$ die Auszeichnung für das , innovativste Produkt“ in der Dermatologie. Das Präparat basiert auf dem Vitamin-ADerivat Alitretinoin und wird zur Behandlung von schweren chronischen Handekzemen eingesetzt. Sales-Direktorin Kerstin Drinnenberg nahm die Auszeichnung bei einem Festakts in München entgegen.

Die Gewinner werden seit elf Jahren in einer unabhängigen Meinungsumfrage in verschiedenen Facharztgruppen ermittelt. Das diesjährige Ergebnis spiegelt die ungestützte Einschätzung von 150 Dermatologen wider. Mit 20\% Spontannennungen führte Toctino ${ }^{\oplus}$ die Rangliste innovativer Therapeutika bei dieser Fachgruppe an. Als Grund für ihre Wahl gaben die Befragten vor allem Wirkvorteile, den spezifischen neuen Therapieansatz, das innovative Therapieprinzip und die gute Verträglichkeit an. Der Hersteller punktete überdies mit der Fachkompetenz und Servicequalität des Außendienste sowie der positiven Gesamtwirkung.

Nach Informationen von Basilea Pharmaceutica, München 